С А.Х. МЕЛИКУЛОВ, И.В. ТЕТВАДЗЕ, А.В. СЕРГЕЕВ, Р.К. АБДУЛКАДЫРОВ, 2014

( ) АННАЛЫ АРИТМОЛОГИИ, 2014

УдК 616.126.4-089:616.124/.125-008.311:616.12-089.8-78

DOI: 10.15275/annaritmol.2014.1.7

\title{
АБЛАЦИЯ АТРИОВЕНТРИКУЛЯРНОГО УЗЛА ПОСЛЕ ВЫПОЛНЕННОЙ ПАРАНОДАЛЬНОЙ АБЛАЦИИ ПРЕДСЕРДНО-ЖЕЛУДОЧКОВОГО УЗЛА ДЛЯ ЛЕЧЕНИЯ ПАРОКСИЗМАЛЬНОЙ ПРЕДСЕРДНО-ЖЕЛУДОЧКОВОЙ УЗЛОВОЙ РИЕНТРИ ТАХИКАРДИИ В УСЛОВИЯХ ИСКУССТВЕННОГО КРОВООБРАЩЕНИЯ
}

Тип статьи: клинический случай

\author{
А.Х. Меликулов, И.В. Тетвадзе, А.В. Сергеев, Р.К. Абдулкадыров \\ ФГБУ «Научный центр сердечно-сосудистой хирургии им. А.Н. Бакулева» \\ (директор - академик РАН и РАМН Л.А. Бокерия) РАМН; Рублевское шоссе, 135, Москва, 121552, \\ Российская Федерация
}

Меликулов Азиз Холмурадович, доктор мед. наук, заведующий лабораторией;

Тетвадзе Инга Владимировна, канд. мед. наук, ст. научн. сотр., главврач Института кардиохирургии им. В.И. Бураковского;

Сергеев Алексей Викторович, канд. мед. наук, научн. сотр., e-mail: sergeev_av@list.ru;

Абдулкадыров Раушан Кадырович, ординатор

\begin{abstract}
В представленном клиническом наблюдении приведены данные пациентки, у которой ранее была выполнена паранодальная аблация предсердно-желудочкового соединения для лечения атриовентрикулярной (AB) узловой риентри тахикардии. Пациентке по поводу пароксизмальной формы трепетания предсердий выполнена радиочастотная аблация правого нижнего перешейка. Диагностирован синдром слабости синусного узла, персистирующая форма фибрилляции предсердий. Далее имплантирован двухкамерный электрокардиостимулятор. Антиаритмическая терапия по поводу персистирующей формы фибрилляции предсердий без эффекта. Принято решение о выполнении радиочастотной модификации предсердно-желудочкового соединения, которую доступом из правого желудочка выполнить не удалось по причине отсутствия верификации спайка пучка Гиса. Доступом ретроградно через аорту удалось создать АВ-блокаду ІІІ степени. Принимая во внимания тот факт, что в 1990-х годах пациентам с атриовентрикулярной узловой риентри тахикардией выполнялась операция паранодальной аблация АВ-узла в условиях искусственного кровообращения, данный клинический случай представляет практическую значимость при катетерной эндовазальной модификации АВ-узлового проведения у данной категории пациентов.

Ключевые слова: атриовентрикулярная узловая риентри тахикардия; паранодальная аблация; радиочастотная аблация.
\end{abstract}

\section{ABLATION OF THE ATRIOVENTRICULAR NODE EXECUTED AFTER PARANODAL ABLATION OF THE ATRIOVENTRICULAR NODE FOR THE TREATMENT OF PAROXYSMAL ATRIAL-VENTRICULAR NODE OF REENTRY TACHYCARDIA IN CONDITIONS OF ARTIFICIAL BLOOD CIRCULATION}

\author{
A.Kh. Melikulov, I.V. Tetvadze, A.V. Sergeev, R.K. Abdulkadyrov
}

A.N. Bakoulev Scientific Center for Cardiovascular Surgery of Russian Academy of Medical Sciences; Rublevskoe shosse, 135, Moscow, 121552, Russian Federation 
Melikulov Aziz Kholmuradovich, MD, DM, Chief of Laboratory;

Tetvadze Inga Vladimirovna, MD, PhD, Senior Research Associate, Head Physician of V.I. Bourakovsky Institute of Cardiac Surgery;

Sergeev Aleksey Viktorovich, MD, PhD, Research Associate, e-mail: sergeev_av@list.ru;

Abdulkadyrov Raushan Kadyrovich, Resident Physician

\begin{abstract}
In this clinical observation is shown the data of the patient who was previously undergone paranodal ablation of atrial-ventricular junction for the treatment of atrioventricular (AV) nodal reentrant tachycardia. Radiofrequency ablation of right lower isthmus for treatment of the paroxysmal form of atrial flutter was made for the patient. Sick sinus node syndrome and paroxysmal form of atrial fibrillation were diagnosed. Then dual-chamber pacemaker was implanted. Antiarrhythmic therapy about the persistent form of atrial fibrillation had no effect. The decision for the implementation of radio frequency modification of atrioventricular connection using right ventriclar access failed because of the lack of verification of the His bundle's spike. Using retrograde access through the aorta we managed to create AV blockade of III degree. Taking into account the fact that in 1990-ies patients with atrioventricular nodal reentrant tachycardia were operated using paranodal ablation of the AV node using extracorporeal circulation, this case has a practical significance when endovascular catheter modification of AV nodal conduction in this category of patients is made. Key words: atrioventricular nodal reentry tachycardia; paranodal ablation; radiofrequency ablation.
\end{abstract}

$\mathrm{A}$ триовентрикулярная узловая риентри тахикардия (АВУРТ) является наиболее часто встречающейся формой наджелудочковой тахикардии и составляет около $50 \%$ случаев всех форм наджелудочковых тахикардий. Аритмия наиболее часто встречается у женщин в возрасте до 40 лет. Средний возраст женщин с АВУРТ составляет 28 лет [1].

G.K. Мое и соавт. впервые описали тот факт, что в основе АВУРТ лежит продольная диссоциация внутриузлового проведения на два пути. Данные авторы также первыми показали, что предсердные экстрастимулы могли индуцировать и купировать АВУРТ у кроликов. В результате проведенных экспериментов было предположено наличие двойных путей проведения в атриовентрикулярном (АВ) узле, при этом $\alpha$-путь имеет более медленное проведение, но короткий рефрактерный период, а $\beta$-путь обладает быстрым проведением, но большим рефрактерным периодом [2].

При синусовом ритме импульс проходит по быстро проводящему $\beta$-пути, что на электрокардиограмме (ЭКГ) проявляется возникновением комплекса $Q R S$. При этом одновременно проводится вниз по медленному $\alpha$-пути и достигает нижней части АВ-узла вскоре после того, как он был деполяризован, и находится в состоянии рефрактерности за счет импульса, проведенного по быстрому пути. При появлении предсердной экстрасистолы импульс блокируется в $\beta$-пути за счет большей рефрактерности последнего и медленно проводится вниз по $\alpha$-пути, что приводит к увеличению продолжительности интервала $P-Q$. Если проведение достаточно медленно для того, чтобы восстановилось проведение по $\beta$-пути, то возникает внутриузловая риентри тахикардия. При более ранней предсердной экстрасистоле вследствие увеличенного времени антеградного проведения $\alpha$-путь имеет больше времени для восстановления проведения, что приводит к индукции устойчивой тахикардии.

Типичная форма АВУРТ по типу slow-fast встречается у 85\% пациентов. Также известны и другие формы тахикардии: fast-slow и slowslow. В целом принято считать, что основными компонентами для возникновения АВУРТ являются структуры, расположенные в области треугольника Коха. Однако последние данные свидетельствуют о том, что не только эти структуры участвуют в АВУРТ, но и межпредсердная перегородка, левое предсердие и коронарный синус [3].

Первые сведения о хирургическом лечении АВУРТ, заключавшемся в создании полной поперечной блокады, были опубликованы S. Gianelli и соавт. в 1967 г. [4]. Авторы предложили выполнять разрез в области АВ-узла при операции в условиях искусственного кровообращения. L. Harrison и соавт. в 1977 г. предложили методику криодеструкции пучка Гиса [5].

Одним из пионеров по использованию метода криодеструкции АВ-узла без применения искусственного кровообращения является Ю.Ю. Бредикис. В НЦССХ им. А.Н. Бакулева под руководством Л.А. Бокерия была разработана методика лазерной перинодальной деструкции у пациентов с АВУРТ [6].

Тринадцатого августа 1982 г. J.L. Cox и соавт. провели первую успешную операцию криоаблации перинодальных структур для лечения АВУРТ [7].

Хирургическая изоляция области предсердно-желудочкового соединения методом много- 
кратной дискретной криодеструкции широко проводилась с 1984 г. Изоляцию осуществляли на работающем сердце с помощью криодеструктора в условиях нормотермического искусственного кровообращения. Определяли исходную антеградную и ретроградную проводимость через AB-узел, производили эпикардиальное картирование предсердно-желудочковой борозды со стороны предсердия на приступе тахикардии или желудочковой стимуляции. В условиях нормотермического искусственного кровообращения после вскрытия правого предсердия выполняли многоканальное эндокардиальное картирование области предсердно-желудочкового соединения с целью определения области раннего ретроградного прорыва возбуждения. По результатам картирования выполнялась серия криодеструкций. Для этой цели использовали криодеструктор с наконечником диаметром 3 мм. Серия деструкций начиналась от верхнего края устья коронарного синуса по переходной зоне предсердно-желудочкового соединения к мембранозной перегородке (рис. 1) [7]. Вторая серия криодеструкций проводилась вдоль нижнего края области предсердно-желудочкового соединения по фиброзному кольцу трикуспидального клапана. Операция заканчивалась криодеструкцией перед устьем коронарного синуса. Криодеструкция выполнялась под постоянным электрофизиологическим контролем интервала $P-Q$ на ЭКГ, интервала $A-H$ на гис- тограмме при температуре $-60{ }^{\circ} \mathrm{C}$ в течение 30-90 с до возникновения преходящей АВ-блокады III степени. Далее производился электрофизиологический контроль эффективности оперативного вмешательства по изменению антеградной и ретроградной проводимости, отсутствию двойных путей АВ-узлового проведения и невозможности индукции узловой тахикардии [8].

При обсуждении операции перинодальной криодеструкции необходимо рассмотреть структурно-гистологические изменения тканей в области предсердно-желудочкового соединения. W.L. Holman и соавт. приводят экспериментальные данные операций на 12 собаках, которым проводили открытую операцию криоаблации перинодальных структур с дальнейшим гистологическим исследованием тканей в области предсердно-желудочкового соединения. Результатом и ключевым моментом данного исследования являлся тот факт, что у 4 (30\%) собак зона воздействия криоаблации в ранний послеоперационный период увеличивалась и распространялась на структуры АВ-узла с возникновением АВ-блокад различных степеней [9].

Необходимо отметить, что в настоящее время стали встречаться пациенты после хирургического лечения АВУРТ, поэтому приобретают важность аспекты интервенционного лечения аритмий у пациентов после подобных операций

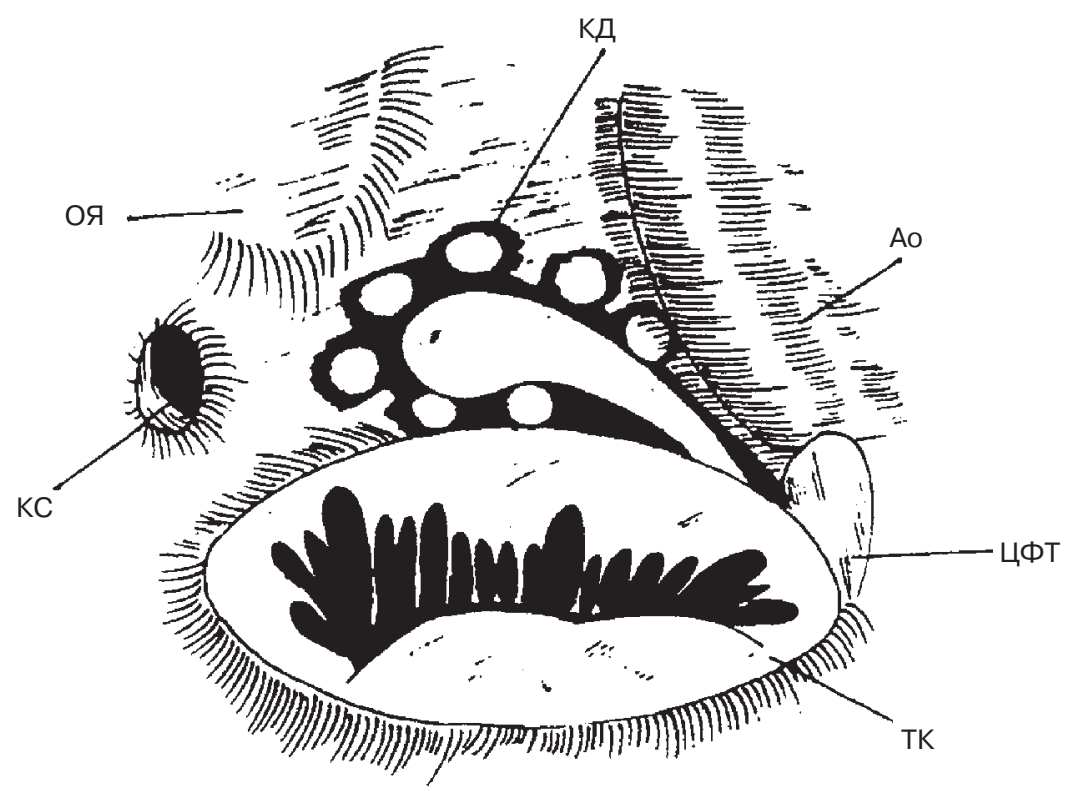

Рис. 1. Схематическое изображение паранодальной криодеструкции.

ОЯ - овальная ямка; Ао - аорта; КС - коронарный синус; ТК - трикуспидальный клапан; ЦФТ - центральное фиброзное тело; КД - криодеструкция 
на открытом сердце. В связи с этим представляем наш клинический случай.

Пациентка 3., 64 лет, поступила с жалобами на общую слабость, головокружение, повышение артериального давления максимально до 180/100 мм рт. ст., приступы учащенного неритмичного сердцебиения.

Считает себя больной с 1988 г., когда впервые возник приступ учащенного ритмичного сердцебиения по данным ЭКГ. Диагностирована типичная АВУРТ, по поводу которой пациентка получала консервативную терапию. Однако консервативная терапия была неэффективной, пароксизмы тахикардии рецидивировали, и 22.01.1993 г. пациентке была выполнена паранодальная аблация АВ-узла в условиях искусственного кровообращения, протокола операции на руках пациентка не имеет. Послеоперационный период протекал без особенностей. Далее пароксизмы тахикардии не рецидивировали.

В последнее время пациентка отмечает ухудшение состояния в связи с развитием пароксизмальной формы фибрилляции и трепетания предсердий. Антиаритмическая терапия с переменным эффектом. Принято решение о проведении электрофизиологического исследования. Антиаритмические препараты отменены за 5 периодов полувыведения. Пациентка доставлена в рентгеноперационную. По общепринятой методике проведены пункции правой бедренной и левой подключичной вен. В полость сердца проведены три электрода для электрофизиологического исследования и установлены в коронарный синус, верхушку правого желудочка и область пучка Гиса. Антеградное проведение по АВ-узлу. Антеградная точка Венкебаха 400 мс. Данных, свидетельствующих о двойных путях проведения через АВ-узел, нет. Антеградный эффективный рефрактерный период АВУ 300 мс (узловые «эхоответы» не регистрируются). Эффективный рефрактерный период левого предсердия 240 мс, ретроградное проведение по АВУ. Ретроградная точка Венкебаха 600 мс, ретроградный эффективный рефрактерный период АВУ 480 мс. Максимальное корригированное время восстановления функции синусного узла 650 мс. При проведении стимуляции коронарного синуса с длительностью цикла индуцировано трепетание предсердий с правым фронтом и длительностью цикла 240 мс. Выполнена радиочастотная аблация правого нижнего перешейка. Время проведения через кавотрикуспидальный перешеек составило 180 и 170 мс соответственно. Далее трепетание предсердий методами учащающей и программированной стимуляции индуцировать не удалось. В связи с наличием признаков синдрома слабости синусного узла рекомендована имплантация двухкамерного электрокардиостимулятора, и 30.01.2013 г. имплантирован двухкамерный электрокардиостимулятор (ЭКС) «Evia DR-T» фирмы «BIOTRONIK».

В послеоперационном периоде проводился подбор антиаритмической терапии с достижением максимально эффективных доз препаратов. Несмотря на проводимое лечение, регистрируются частые пароксизмы фибрилляции предсердий, плохо переносимые пациенткой. Рекомендована модификация АВ-узлового проведения, что входит в рамки стратегии «ablate and pace» («аблация и стимуляция»).

\section{Операция}

Пациентка доставлена в рентгеноперационную на ритме от ЭКС в режиме DDD с базовой частотой 70 уд/мин; ЭКС переведен в режим VVI с частотой 40 уд/мин. Под комбинированной анестезией пунктирована правая бедренная вена. В полость сердца проведен управляемый орошаемый аблационный электрод BW Celsius ThermoCool и установлен в среднесептальную область, однако спайк пучка Гиса верифицировать не удалось, несмотря на тщательно проведенное картирование. Пробные радиочастотные воздействия без эффекта, при этом медленный или ускоренный узловой ритмы не развиваются. Пунктирована левая бедренная артерия, в полость левого желудочка проведен управляемый аблационный электрод Medtronic Marinr MC и установлен в левой среднесептальной области (рис. 2). Однако и в данной области спайк пучка Гиса не верифицирован (рис. 3). Далее проведено пробное радиочастотное воздействие (мощность 30 Вт, температура $40-45{ }^{\circ} \mathrm{C}$, сопротивление 95-110 ом) длительностью 40 с в левой среднесептальной области с развитием быстрого узлового ритма и АВ-блокады III степени с частотой сокращений желудочков менее 30 уд/мин. На этом процедура завершена. Через 1 сутки при интеррогировании с ЭКС для проверки AB-проведения выявлено, что АВ-блокада III степени сохраняется. Пациентка выписана в удовлетворительном состоянии. 


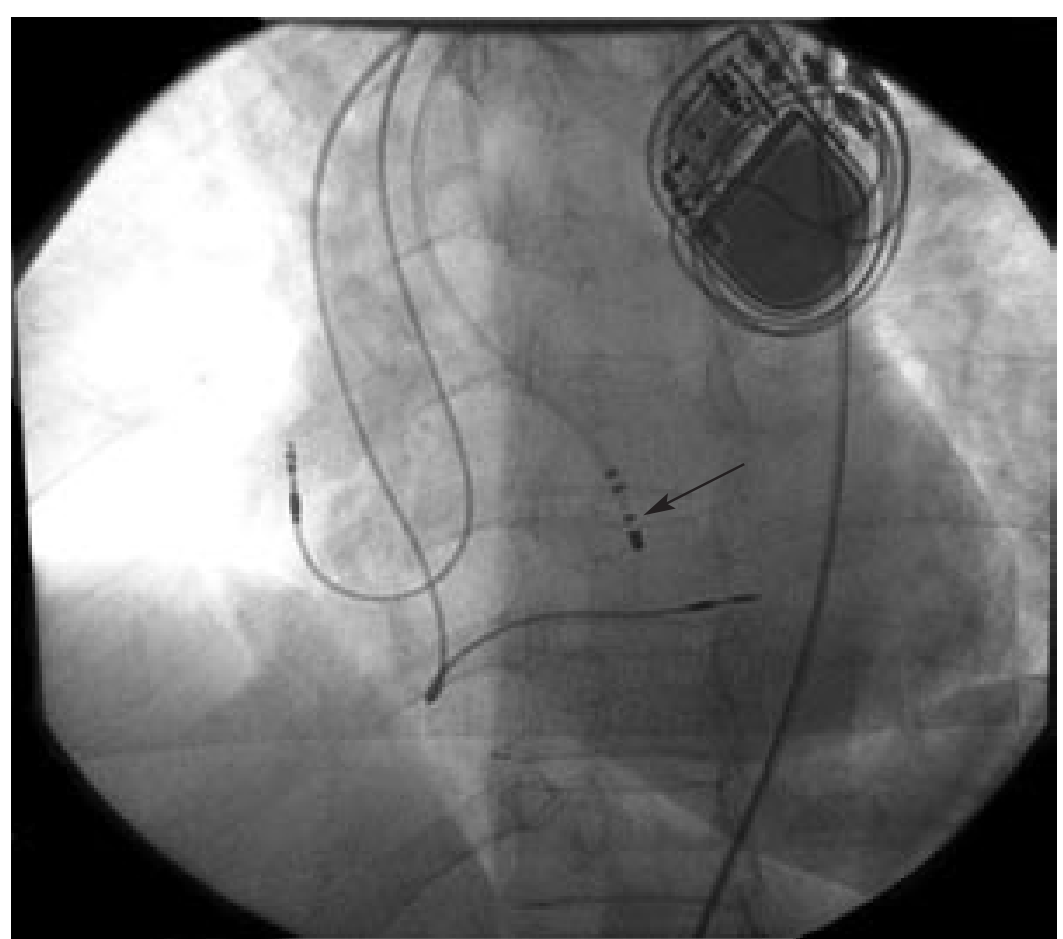

Рис. 2. Интраоперационная рентгеноскопия (проекция LAO 30). Аблационный картирующий катетер в левой среднесептальной области, проведенный трансаортально, указан стрелкой

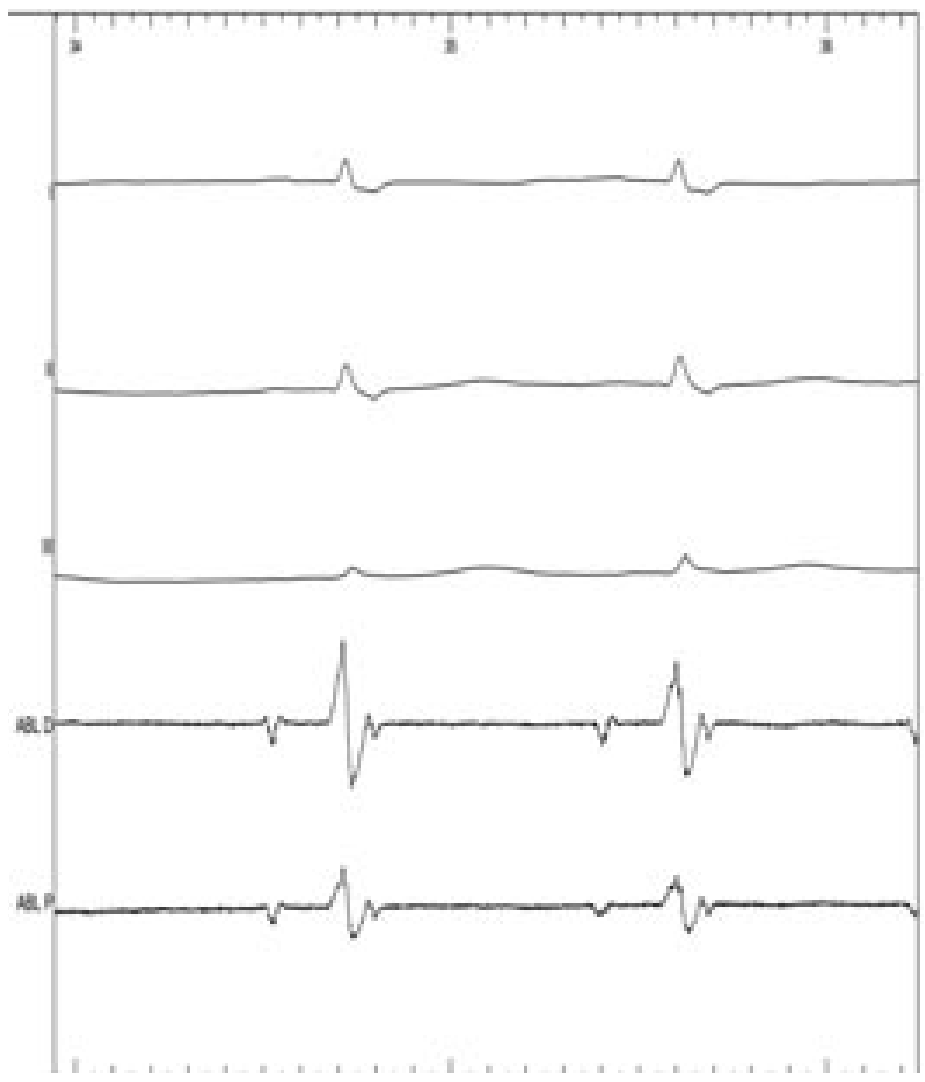

Рис. 3. Интраоперационные эндограммы. Картирующий электрод, проведенный трансаортально, расположен в левой среднесептальной области, при этом спайк пучка Гиса не верифицируется 


\section{Заключение}

Принимая во внимание тот факт, что в 1990-х годах пациентам с АВУРТ выполнялась операция паранодальной аблации АВ-узла в условиях искусственного кровообращения, данный клинический случай представляет практическую значимость при катетерной эндовазальной модификации АВ-узлового проведения у данной категории пациентов.

Мы предполагаем, основываясь на экспериментальных данных, приведенных в 1988 г. W.L. Holman и coaвт. [9], что причиной неэффективности радиочастотной модификации АВ-узлового проведения доступом из правого желудочка может служить то, что при выполненной паранодальной криодеструкции на открытом сердце образуется фиброзная ткань, которая препятствует проникновению радиочастотной энергии при дальнейшей катетерной модификации АВ-узлового проведения.

Данный факт, несомненно, необходимо принимать во внимание при проведении интервенционных процедур у данной группы пациентов.

\section{Конфликт интересов}

Конфликт интересов не заявляется.

\section{Библиографический список}

1. Bashir Y., Betts T.R., Rajappan K. Cardiac electrophysiology and catheter ablation. Oxford University Press; 2010.

2. Issa Z., Miller J.M., Zipes D.P. Clinical arrhythmology and electrophysiology. WB Saunders; 2012.

3. Ho R.T. Electrophysiology of arrhythmias: practical images for diagnosis and ablation. Lippincott Williams and Wilkins; 2010.
4. Gianelli S., Jr, Ayres S.M., Gromprecht R.F. et al. Therapeutic surgical division of the human conduction system. JAMA. 1967; 199 (3): 155-60. DOI: 10.1001/jama.1967.03120030059008.

5. Harrison L., Gallagher J.J., Kassell J. et al. Cryosurgical ablation of the A-V node-His bundle. A new method for producing A-V block. Circulation. 1977; 55 (3): 463-70. DOI: 10.1161/01.CIR.55.3.463.

6. Бокерия Л.А. Тахиаритмии. Диагностика и хирургическое лечение. Л.; 1989.

7. Cox J.L., Ferguson T.B., Jr, Lindsay B.D. et al. Perinodal cryosurgery for atrioventricular node reentry tachycardia in 23 patients. J. Thorac. Cardiovasc. Surg. 1990; 99 (3): 440-9; discussion 449-50.

8. Базаев В.А. Диагностика и результаты хирургического лечения атриовентрикулярной узловой риентри тахикардии. Дис. ... канд. мед. наук. М.; 1994.

9. Holman W.L., Hackel D.B., Lease J.G. Cryosurgical ablation of atrioventricular nodal reentry: histologic localization of the proximal common pathway. Circulation. 1988; 77: 1356-62. DOI: 10.1161/01.CIR.77.6.1356.

\section{References}

1. Bashir Y., Betts T.R., Rajappan K. Cardiac electrophysiology and catheter ablation. Oxford University Press; 2010.

2. Issa Z., Miller J.M., Zipes D.P. Clinical arrhythmology and electrophysiology. WB Saunders; 2012.

3. Ho R.T. Electrophysiology of arrhythmias: practical images for diagnosis and ablation. Lippincott Williams and Wilkins; 2010.

4. Gianelli S., Jr, Ayres S.M., Gromprecht R.F. et al. Therapeutic surgical division of the human conduction system. JAMA. 1967; 199 (3): 155-60. DOI: 10.1001/jama.1967.03120030059008.

5. Harrison L., Gallagher J.J., Kassell J. et al. Cryosurgical ablation of the A-V node-His bundle. A new method for producing A-V block. Circulation. 1977; 55 (3): 463-70. DOI: 10.1161/01.CIR.55.3.463.

6. Bockeria L.A. Tachyarrhythmias. Diagnosis and treatment. Leningrad; 1989 (in Russian).

7. Cox J.L., Ferguson T.B., Jr, Lindsay B.D. et al. Perinodal cryosurgery for atrioventricular node reentry tachycardia in 23 patients. J. Thorac. Cardiovasc. Surg. 1990; 99 (3): 440-9; discussion 449-50.

8. Bazaev V.A. Diagnosis and results of surgical treatment for atrioventricular nodal reentry tachycardia. Med. Sci. Diss. Moscow; 1994 (in Russian).

9. Holman W.L., Hackel D.B., Lease J.G. Cryosurgical ablation of atrioventricular nodal reentry: histologic localization of the proximal common pathway. Circulation. 1988; 77: 1356-62. DOI: 10.1161/01.CIR.77.6.1356.

Поступила 26.05.2014 г.

Подписана в печать 20.07.2014 г. 